\title{
Increasing user engagement on blockchain applications through persuasive design
}

\author{
Manuel Knott \\ University Passau \\ Chair of Management, People and \\ Information \\ $\underline{\text { manuel@prolabart.com }}$
}

\author{
Anne-Sophie Mayer \\ University Passau \\ Chair of Management, People and \\ Information \\ anne-sophie.mayer@uni-passau.de \\ Marina Fiedler \\ University Passau \\ Chair for Human Resource \\ Management \& Intrapreneurship \\ fiedler@lmpi
}

\author{
Franz Strich \\ University Bayreuth \\ Chair for Human Resource \\ Management \& Intrapreneurship \\ franz.strich@uni-bayreuth.de
}

\begin{abstract}
Blockchain gives rise to many new applications and use cases and has already markedly changed several industries, such as financial services, energy and utilities, or healthcare. Although blockchain could potentially be used disruptively for end-user applications as well, utilizing it remains poor. It appears that the unconvincing design of many end-user blockchain applications leads to insufficient user engagement. To investigate the influence of design aspects on users' engagement of blockchain end-user applications, we developed a blockchain application for the creative industries based on the principles of persuasive design. Hereby, we aim to contribute to research in the blockchain context on how end-user applications need to be designed to increase user engagement. By using a design science research process, we can ultimately provide a total of seven recommendations for developing persuasive blockchain applications for end-users.
\end{abstract}

\section{Introduction}

Blockchain is one of the most promising technologies of the future which has already given rise to many disruptive applications and use cases [1]. While the general interest in blockchain technology is steadily increasing [2], the widespread use in end-user applications is surprisingly underdeveloped. To date only a number of niche products and services for end-users rely on blockchain technology [3]. This is surprising considering the tremendous progress this technology has made in the past few years [4]. It seems that the perceived underlying complexity of blockchain and difficulties in handling existing applications prohibit users from engaging with the technology [5].
Despite the promising technological advantages blockchain has over centralized applications, recent uses cases in the creative industries provide further evidence that blockchain applications' design, does not allow for sufficient user engagement $[5,6]$. For instance, the music streaming platform UJO-Music follows a business model on the blockchain, which allows royalty payments automatically to be paid out after a song has been streamed. In this way, by relying on blockchain technology, UJO-Music manages to shorten the period between a user's music consumption and royalty payments to the artists from several months or years to a few seconds [7]. Nevertheless, since its launch in 2015, UJO Music has not managed to tap into a mass market. In contrast, between 2015 and 2020, Spotify has increased its number of monthly active users from 91 million listeners to 345 million [8]. In the meantime, UJO Music's service has become unavailable. In trying to determine the reasons underlying this ambivalent market position of both suppliers, Spotify's extensive utilization of persuasive design principles becomes apparent as a main distinguishing factor between the two platforms $[9,10]$.

The principles of persuasive design focus on changing users' behavior through persuasion and social influence [5]. Persuasive design provides a comprehensive framework for successfully designing applications that can facilitate user engagement [11]. Spotify, AirBnB, Tinder, or Netflix are prominent examples of persuasive design principles in end-user applications being successfully realized. All of these applications benefit significantly from a steadily high user engagement which results from utilizing persuasive design [9]. Blockchain applications, can widely benefit from persuasive design as well; however, at least in our understanding, no blockchain application has applied the 
principles of persuasive design in an all-encompassing manner yet. Our assumption about the missing consideration of persuasive design principles in the development of blockchain applications was supported by qualitative interviews we conducted with 35 professionals in the creative industries who utilize blockchain applications in their daily work.

We therefore developed an end-user blockchain application for the creative industries using the principles of persuasive design. This custom blockchain application is designed for professionals in the creative industries to automate formal processes such as contracting and remuneration. The creative industries provide a perfect environment to explore the realization of persuasive design principles on blockchain applications. On the one hand, blockchain offers quite a few benefits for professionals in the creative industries, giving the necessary intrinsic motivation to at least try our application. On the other hand, the blockchain's predominantly project-based activities and the accompanying recurring formal processes lead to the app being highly utilized. Therefore, we believe that our use case provides the foundation for a necessary and promising research direction for the impact of persuasive designs on blockchain applications and their user engagement.

Our paper is structured as follows: First, we address the potential blockchain technology holds for the creative industries. Then, we present the persuasive design framework. Next, we present our design science research (DSR) approach. Building on this, we make recommendations for future blockchain application development, taking our experience with persuasive design into account. Lastly, we end the paper with a brief conclusion.

\section{Blockchain technology's untapped po- tential in the creative industries}

The creative industries are the leading drivers of innovations and can widely benefit from blockchain technology [12]. These industries encompass a wide set of concerns, including sectors like advertising, architecture, film-production, or software development [13]. The range of industries are generally characterized by a project-based organizational structure [14]. With their predominantly project-based organization and intellectual property as the core of their value creation, creative industries can benefit from blockchain in several ways [12]. First, formal processes such as contracting or team finding can be automated by blockchain using automatic contracts, so-called smart contracts. Here, transactions can be executed efficiently without having to rely on third parties, as trust in middlemen such as lawyers or banks is replaced by trust in technology $[15,16]$. Second, intellectual property rights, whether physical or digital intellectual property, can be stored efficiently and cost-effectively on the blockchain, for example in the form of so-called non-fungible tokens (NFTs) [17]. Third, transaction costs can be drastically reduced in intellectual property sales on the blockchain, as trusted intermediaries between buyer and seller, who act as third parties, become redundant [18]. Despite these promising possibilities of blockchain for creative industries, widespread use of the technology remains unfulfilled.

\section{Increasing user engagement through persuasive design}

Persuasive design provides a framework for developing user-oriented and persuasive information systems (ISs). Persuasive design aims to "form, alter or reinforce attitudes, behaviors, or an action" [23, p. 1225] towards an IS without coercion or deception. It therefore seeks to induce behavioral change through technology interaction without compulsion [20]. For example, behavioral change can be seen as the change occurring when users transfer from not using blockchain-based applications to using them. Ideally, the concept of persuasive design achieves a cognitive or emotional transformation of a certain mental state toward a specific action $[9,21]$. Fundamentally, behavioral change requires individuals who are (1) sufficiently motivated, (2) able to change their behavior, and (3) have a sufficient trigger for changing their behavior [5].

First, individuals must be sufficiently motivated to change their behavior. Fogg [5] identified three twosided motivators that function in this way, which are pleasure/pain, hope/fear, and social acceptance/rejection. The feeling of hunger that needs to be satisfied, for example, represents pain and can provide sufficient motivation [20]. In the specific use case of blockchain end-user applications, a possible pleasure could be the possibility of streaming digital content without country-specific restrictions. Next, the hope/fear motivator type is preceded by an expectation that something good (or bad) will happen as a result of a behavioral change $[5,20]$. Finally, the motivator pair social acceptance/rejection relates to a behavioral change that depends on an expected societal reaction $[5,10]$. Nevertheless, being motivated on its own is not enough. At the same time, the individual must have the appropriate ability to change their behavior [5].

Therefore, second, an IS must by design be simple enough to maintain the necessary abilities for behavioral change [19]. Systems that require the user to learn new skills to use them correctly usually fail due to user resilience. Fogg refers to simplicity as a "function of a 
person's scarcest resource at the moment a behavior is triggered" [8, p.7]. Thus, the aim cannot be to help users increase their ability to use an IS. Rather, ISs must have low complexity, even intuitively relying on the power of simplicity [11, 19]. Fogg [5] asserts that a system is simple only if it simultaneously requires a low level of effort regarding time, monetary resources, physical features, and cognitive input. Additionally, an IS should not deviate from social norms and routine activities [22]. End-user applications should therefore be intuitive to use and specifically align with the enduser's routines. Yet, in addition to sufficient motivation and requisite abilities, behavioral change will not occur without a suitable trigger.

Thus, third, if individuals are sufficiently motivated and able to change their behavior, a particular trigger can ultimately lead to the desired behavioral change. To appropriately prompt users to change their behavior, there are three types of triggers: sparks, facilitators, and signals [5]. First, sparks use one of the three two-way motivators (i.e., pleasure/pain, hope/fear, social acceptance/rejection) as an initiator. Sparks can, for example, be texts that evoke fear or videos that convey hope $[5,23]$. Second, facilitators can be decisive triggers if individuals lack motivation. For instance, one-click purchase options that indicate a desired behavior is easy to achieve [5], can facilitate change. Finally, signals can serve as reminders for an individual to change behavior if motivation and ability are already sufficiently present $[5,24]$. Thus, as triggers, signals do not motivate or simplify, but rather like a green light - indicate to an individual that a given behavior can now be changed [20, 21]. However, not the mere presence of the triggers, but rather the interplay of sufficient motivation, the necessary ability, and a suitable trigger can lead to a behavioral change.

To successfully change users' behavior, the three factors (motivation, ability and trigger) must occur simultaneously and without coercion [5, 11]. Fogg [5] further argues that systems achieve faster behavioral change by focusing less on motivating users and more on making the desired behavior easier.

In sum, persuasive design helps to design compelling ISs that induce a desired change in the user's behavior. This framework also provides the necessary tools to identify problems that prevent a desired behavior in a system. For this purpose, persuasive design combines technological design principles with insights from behavioral psychology [19]. Combining the two fields enables unconstrained aspirations for a change in behavior, based on users' own fundamental convictions [25]. Thus, persuasive design offers an excellent framework for motivating users to change their behavior regarding blockchain technology.

\section{A DSRP for persuasive design in block- chain applications}

A design science research approach enabled us to generate sound knowledge for academia and practice on novel solution design in the IS domain [26]. These novel solutions, called artifacts in the DSR context, can include new kinds of software, methods, models, or concepts [27]. In our case, relying on DSR, we structurally investigated how persuasive design impacts user engagement of blockchain applications. The design knowledge gained through DSR can help researchers in future projects to design artifacts in a systematic and scientifically sound manner [26].This requires not only describing the design of the artefacts while conducting a DSR, but also investigating its broad impact on the application domain [28]. This specific research approach allows us to holistically discuss the effective use of artifacts in the real world, as well as their impact and influence in scientific theory, specifically in using a design science research process (DSRP) [27].

The DSRP provides a process model for the design and development of IT artifacts in the IS community [29]. It is based on six activities, which nominally follow a sequence, even if not always stringently: (1) problem identification and motivation, (2) objectives of a solution, (3) design and development, (4) demonstration, (5) evaluation, and (6) communication [29]. The entry point for research that uses a DSRP can be of various origins. Besides a problem-centered approach, other possibilities are an object-centered solution or a design-and-development centered approach [26, 28]. A problem-centered approach recommends starting with the problem identification and motivation, insofar as the idea for the IT artifact evolves from previous research or observation [27, 29]. Since the idea for our IT artifact emerged from qualitative interviews, we begin our DSRP with the problem identification and motivation, using the problem-centered approach as our entry point.

\subsection{Problem centered approach}

We used a qualitative approach to find out why blockchain applications are insufficiently used in the creative industries. We conducted 35 semi-structured interviews with professionals in the creative industries who are using blockchain applications. An overview of our interview sample is given in Table 1. 


\begin{tabular}{|l|c|c|c|c|c|}
\hline \multicolumn{7}{|c|}{ Table 1: Overview of the survey sample } \\
\hline Industry & $\#$ & $\boldsymbol{\varnothing}$ age in years & $\boldsymbol{\varnothing}$ work experience & Gender $\boldsymbol{f} / \boldsymbol{m}$ & Nationalities \\
\hline Art & 7 & 38 & 11 & $3 / 4$ & DE, MX, PT, US \\
\hline Design & 1 & 26 & 8 & $0 / 1$ & US \\
\hline Fashion & 3 & 41 & 15 & $1 / 2$ & AR, PAT, US \\
\hline Music & 6 & 27 & 5 & $2 / 4$ & BE, DE, SP, US \\
\hline Photography & 8 & 32 & 7 & $0 / 7$ & DE, MX, PL, US \\
\hline Software & 7 & 34 & 6 & $0 / 7$ & EN, RO, US \\
\hline Video and Film & 3 & 39 & 9 & $0 / 3$ & DE, EN, SL \\
\hline $\begin{array}{l}\text { Note: AR = Argentina; BE = Belgium; DE = Germany; EN = England; MX = Mexico; PAT = Patagonia; } \\
\text { PL = Poland; PT = Portugal; RO =Romania; SL = Slovenia; US = United States of America }\end{array}$ \\
\hline
\end{tabular}

Our questions focused on desired adjustments of blockchain applications, and on challenges in adopting blockchain technology. This allowed us to gain meaningful insight on what enhancements current blockchain solutions need to suitably engage the creative industries, and on what is currently preventing sufficient blockchain technology use. We coded the transcribed interviews and analyzed them according to Webster and Watson [30].

Professionals in the creative industries complained about many blockchain applications lacking user-centricity. In particular, users missed much needed flexibility they require on the platforms to perform their primary tasks adequately:

\section{"But at the same time, you have hundreds of photographers ap- plying for the same shoot, you cannot explain anything about yourself. So, they can only rely on the photos they receive from you, but on the other platforms \\ I can write why I am the best photographer for the job, and so on." (Artist, Photography)}

In addition, interviewees consistently criticized blockchain applications for providing user interfaces that are hardly intuitive. As a result, many find it difficult to use these applications comprehensively and efficiently:

"So, I felt from my point of view this site doesn't work. Yeah, you know, no matter what item they are asking or whatever I have to do, I don't know how it works. I don't know, the visual platform you kind of need to

have a professional background

to use it." (Artist, Music)

Further, participants criticized the User Interface and User Experience (UI/UX) of different platforms. Especially, they mentioned the lack of design aesthetics:

"This display for the users, you know, the user experience here is really rough." (Artist, Film, Video \& TV)

When blockchain applications integrated payment functions, they were often criticized for being too inflexible and unintuitive. For a blockchain application to rely on a payment mechanism a so-called crypto wallet must be accessed by the application itself. Only then can payments be made on the blockchain. Those wallets serve to manage crypto assets and the necessary keys for sending and receiving crypto currencies [31]. However, many blockchain applications do not satisfactorily implement integrating and handling these wallets:

$$
\begin{gathered}
\text { "Yeah I would want my wallet } \\
\text { to do a bunch of stuff. Yeah. But } \\
\text { once you access your profile } \\
\text { through the wallet and run the } \\
\text { profile, it would be good to } \\
\text { have some statistics to see what } \\
\text { kind of traffic one has." (Artist, } \\
\text { Music) }
\end{gathered}
$$

Therefore, professionals in the creative industries are hopeful that blockchain applications will improve their design in the future:

"Now I think people are going to find better user interfaces for 


\section{it. It makes things more trans- parent and simpler than to use light gray. Currently this a little too difficult to use, and it's diffi- cult for artists to use. You know, just like the old smartphones, you know, till the iPhone came along with an easier interface, you know, people struggled with them." (Artist, Graphic Design)}

Our qualitative survey revealed that common blockchain applications lack a persuasive design. In particular blockchain applications for the creative industries seem to be unintuitive, inflexible, and unappealing. Thus, as the first DSRP step, the lack of user orientation and the associated unconvincing design are the foundations on which we did the problem identification and motivation.

\subsection{Problem Identification and Motivation}

The interviews we conducted revealed that professionals from the creative industries need a persuasive design feature when they use blockchain applications. Our results agree with the assessments research and practice have produced. Arcos [6], for example, has already stated that the functionality of a blockchain should only be embedded at the back end of the software architecture, and in such a way that it does not interfere with the common user experience. This view not only goes hand in hand with our interview results, but also with IBM's [15, p.1, 29] assessment: "Despite all the euphoria about blockchain technology, when designing applications, the focus must be on the human as the user and not on the machine as the technology driver."

Based on the above, we find that many blockchain applications lack a persuasive design, which results in potential users not engaging with those applications due to them missing the necessary abilities and motivation. Therefore, it is important to develop blockchain applications with a persuasive design that focuses on the users and their needs.

\subsection{Objectives of a solution}

To investigate whether a persuasive design can lead to increased user engagement on blockchain applications, we designed ProLabArt according to the persuasive design principles. ProLabArt is a user-centered blockchain-powered project management tool for project-based organizations in the creative industries, which facilitates formal organization and coordination. It provides support for typical organizational tasks in project-based organizations. In particular, ProLabArt automates team management and contracting, by relying on the Ethereum blockchain.

\subsection{Design and development}

ProLabArt brings the Ethereum blockchain and its' cryptocurrency Ether in a native SWIFT programmed iOS app that was developed for mobile devices with an $i O S$ operating system. Firebase is used as the main database. However, for data protection and security reasons, the talents' (i.e. team members) public keys are only locally stored on the devices. Communication with the Ethereum Blockchain is established via a custom node using the Infura API. The Web3Swift API is used as an interface between the Ethereum blockchain and SWIFT. The app is available in the Apple App Store.

Our application has two main motivator functions. First, ProLabArt motivates users to engage with blockchain technology by automating the team management aspect of projects in the creative industries. Usually, project leaders assemble the teams through social networks and informal recruitment. ProLabArt automates these processes by relying on algorithms we developed for this specific purpose. Consequently, ProLabArt relieves project leaders from the tedious and time-consuming task of team management.

Second, ProLabArt motivates users to engage with blockchain by enabling blockchain-powered contracting. For this purpose, all contract-relevant content is automatically stored in a legally binding contract on the Ethereum blockchain. Upon completion of a project, team members can be compensated via cryptocurrency within the app. Through this function we relieve project leaders and team members of the tedious and time-consuming repetitive processes of contracting and properly compensating contract workers by essentially automating them through the Ethereum blockchain.

On considerations of ability, ProLabArt tries to keep the skill requirement for using the app low, to encourage the end-users to positively change their behavior toward blockchain. To support the primary task, content is provided tailored for individual users. For example, information is prepared individually for the project leaders as well as the remaining talents by addressing each user by name. In addition, in the project execution process the user is continuously tunneled through the entire lifecycle of the app. Among other things, functions and areas of the app are only enabled in compliance with a project's lifecycle. For example, 
the payment option is only enabled once the project has officially been completed.

ProLabArt also relies on self-monitoring, giving each user the ability to constantly monitor their progress and assess the performance of their talents. Thereby, we strengthen users' possibility of observing their own potential target behavior, which motivates them toward a potential behavior change. With extensive onboarding, an explanation video, and a graphical representation of ProLabArt's cause-and-effect relationships, we enable users through simulation to see the immediate link between the cause and effect of their potential activity.

Finally, to achieve the primary task, the end users are given the possibility of sufficient practice. First, ProLabArt suggests dummy talents for users to familiarize themselves with the app, and second, when we reward talents with cryptocurrencies, we offer the project leaders the possibility of sending fake cryptocurrency via the Ropsten testnet. Through this, the project leaders can comprehensively familiarize themselves with the app in general, and with blockchain as well as cryptocurrency in particular.

Within ProLabArt we facilitate a computer-human-dialogue by implementing comprehensive dialogue support. Users get text alerts that give positive feedback after each task completion. At the same time, we use text alerts for other communications, e.g., when opening the app, as a reminder function to achieve the primary goal. Therefore, we make users more open to persuasion through praise. In addition, we achieve a high level of dialogue support by giving users the greatest possible freedom in the team selection process. Instead of simply determining a team to collaborate with the project leader, we propose different teams from which the user can ultimately choose. In this way, we increase the app's persuasive power at opportune moments, rather than just determining the team solely through the app. Finally, within the app we also want to embrace our social role toward more diversity, equity, and inclusion (DE\&I) in the creative industries. It was important to us that the app would proactively embrace DE\&I. The app provides no images, information on talents' ethical background, age, religion, etc. Thus, we can avoid stereotypic behavior based on external characteristics. We also communicate our self-image as a role model for more DE\&I in the creative industries in detail and transparently at various points within the app, not least to increase our system's credibility.

To increase our blockchain application's system credibility, we have continuously communicated ProLabArt's academic background. We found it important to comprehensively explain that we do not gain any monetary rewards with the app. We also emphasized that any information we provide is unbiased, truthful and shows expertise. In addition, all available content and information is verifiable and further supported by endorsements from respected third-party sources. We also added a comprehensive "about-us section" in the app, explaining the individual roles of those involved and providing a sufficient real-world feel through background information on the responsibilities of the app. In addition, there are several opportunities for establishing direct contact on a variety of platforms with each of the people responsible for the app. Knowing about the urgency of a high surface credibility, we focus strongly on a holistic competent look and feel from onboarding to the settings area. Finally, we used a cross-platform approach to achieve the highest possible social support through the app.

Through a comprehensive online presence, we can leverage social impact that motivates users for a behavioral change toward blockchain. ProLabArt has an extensive website with background information, motivation, goal, roadmap, and explainer videos about the app and its functionalities. In addition, we implemented a blog on our website to educate readers about blockchain for creative industries. Further, we keep our followers up to date via our own Twitter and Telegram account. Finally, we set up a Discord channel with various subchannels where the community can interact directly with us and with one another. Among others, we opened a subchannel where users can describe their experiences and use cases. In doing so, we want to reinforce social learning by allowing users to observe their own target behavior based on what other users do. Thereby, we intend to increase artists' motivation to perform the primary task. In addition, the website's different channels, and especially the interactive exchange via Discord, allows us to leverage the app's normative influence. Within Discord the communication between users can increase peer pressure on different individuals, increasing their willingness to adopt the target behavior, i.e., extensive use of the blockchain application. Next, we show our approach to generating a sufficient sample, and explain how we analyzed the data.

\subsection{Demonstration}

We used a cross-platform approach to achieve the highest possible outreach for the app. ProLabArt has an extensive website with background information, motivation, goal, roadmap, and explainer videos about the app and its functionalities. In addition, we started a blog on our website that educates about blockchain for creative industries. ProLabArt was made available to interested parties and selected testers as a beta ver- 
sion between September 2020 and January 2021. In total, 19 project leaders gave us feedback using either the various channels such as Twitter, Telegram, Discord or the in-app feedback function. In addition, the app was presented to several UI/UX designers, as well as to product designers with several years of experience. The feedback we collected in this way was exclusively in written form.

\subsection{Evaluation}

The evaluation of the collected feedback was divided into three areas of interest, namely design, team finding, and contracting. First, we evaluated the feedback regarding the design. The testers' feedback showed us that a significant proportion of app users engaged with ProLabArt using iPads. Since our design was primarily focused on iPhone usage, we adapted it for common iPad resolutions. In addition, we improved our UX by eliminating redundant steps and reducing the number of intermediate steps to achieve the primary goal. We also changed some parts of the font for better screen readability.

Second, we addressed the feedback of the team finding system by extending it in significant parts. A fundamental improvement was the function to replace individual team members from a proposed team. This gives users the necessary flexibility in their team configuration in case one of the team members is unable to participate in the project.

Third, feedback from testers indicated that a display of current exchange rates (Ether/USD, Ether/EUR) and the user's cryptocurrency balance within the app can help to reward team members more efficiently and conveniently.

Finally, we received minor improvement suggestions from the Apple Developer Program Support before the app was released in the Apple App Store. These were graphical bumps that occurred on iPhone 8. Specifically, there was an overlap of text content on the onboarding screen. This has also been fixed, so that the app is available to the public for free in the Apple App Store since January 26, 2021.

\subsection{Communication}

We circulated information on the app's official launch and triggered users to engage with ProLabArt via the ProLabArt website, and the associated Twitter, Telegram, and Discord channels. In addition, we contacted 2458 companies from the creative industries in the $\mathrm{DACH}$-region, thus triggering them with a request to use the app.

\section{Recommendations for building persua- sive blockchain applications}

\subsection{Focus on your value proposition}

Users will measure the benefit of an application primarily by its perceived value proposition. The fact that an application relies on blockchain does not on its own provide sufficient motivation to engage with it. Therefore, blockchain has to contribute with the rest of the system to the value proposition, and as Arcos [6] already stated, it should best disappear in the back-end of the software architecture. When communicating blockchain as part of the value proposition, we highly recommend that users do so in a generally understandable and comprehensible way. Many potential users will not fully understand blockchain, which would result in them feeling rather overwhelmed by the app and therefore not motivated to use it. Moreover, users will feel they are insufficiently skilled to use the app properly. The principle of persuasive design, which determines that ISs must be designed simply to fit the necessary skills for behavioral change, equally counts for communicating their value proposition.

\subsection{Not everything has to run on the block- chain}

Blockchain's unique strengths are also its greatest weaknesses. Blockchain is primarily used to achieve immutability, transparency, automation of processes, or to eliminate middlemen in various processes. This is where blockchain technology's full potential lies. However, its immutability makes it almost impossible to react flexibly to different influences and situations. Further, it is virtually impossible to delete data from a blockchain. This can result in legal and financial consequences in the event of data protection violations or security breaches if incorrect data transmission occurs. Furthermore, with blockchain's increasing popularity, the costs per transaction or contract execution are steadily rising. Therefore, only the most necessary information should be stored on the blockchain. Also, blockchain's immutability significantly complicates subsequent changes in the service. However, if a hybrid system of centralized and decentralized data storage is used, the service can be adapted at any time and if at all, only small changes need to be made to the blockchain itself. Then, there are less concerns if the users' motivation or their abilities are not sufficient to use the app comprehensively. With a hybrid system considerable operating costs can be saved if it is not necessary to run every data transfer on the blockchain. 


\subsection{Choose the right blockchain for your use case wisely}

Just because Ethereum is currently the talk of the town doesn't mean it's the best solution for every use case. There are probably hundreds, if not thousands, of different blockchains each with its own strengths and fields of application. Therefore, it makes sense to choose the right blockchain based on requirements rather than on popularity. When we started developing the app, a transaction using our app cost about USD 0.000047. In March 2021, the cost of a transaction peaked at USD 13. The high computational cost and the ever-increasing demand for Ether almost caused our project to fail, because such high costs meant the Ethereum blockchain was no longer suitable for our use case. Users are not motivated to pay unprecedentedly high transaction costs for comparatively small transaction gains. In our case, using the Free TON blockchain platform instead might have been smarter, as it offers millions of transactions per second at a price of less than USD 0.01. However, we were tempted to select Ethereum, the top performer in decentralized applications, without considering other alternatives.

\subsection{Do not re-invent the wheel}

There are many highly talented programmers pioneering blockchain technology and offering strong frameworks, API's, and third-party services that will save a lot of work when developing new blockchain use cases. Ganache, Truffel, Infura, Ropsten, Rinkeby, or web3 are all projects programmers and developers initiated on a voluntary basis to make blockchain applications accessible to a wider audience. These projects rely on a large community that offer extensive support in the form of instructions, tutorials, or forum threats. In our case, we were very grateful to be able to draw on these resources. We are convinced that without such help, the project could not have been realized. There is still a great deal of movement and development in the blockchain world, so we strongly recommend reliance on well-established frameworks, APIs, and third-party libraries to build solid and stable applications. In-house development of already well-developed and tested interfaces and services is usually redundant and therefore not worthwhile, especially since the vast majority of third-party services are free of charge anyway.

\subsection{Costumer support is key to success}

Especially during startup, most of the financial and human resources will go toward $\mathrm{R} \& \mathrm{D}$ and marketing. However, it is at least as important to maintain and cultivate solid customer support services. For many blockchain is what the internet was in the 1990s. Cryptocurrencies, decentralization, or wallets are still unknown to most end-users. The terms and technologies are hardly understood and have yet to be mastered. Therefore, comprehensive customer support will motivate the end-user to continue using the app and can make a significant contribution to end-users' long-term engagement with the application. Specifically, it is useful to provide extensive educational material regarding the application and blockchain technology in the form of videos, blog posts, or tutorials in order to provide users with the necessary abilities to engage with the app. Also, giving users different channels and possibilities to get in contact with a customer care unit or community can greatly increase the customer retention.

\subsection{Be transparent and credible}

Since the emergence of blockchain technology, it has been associated with a flimsy connotation as a technology of the dark net. The fact that in the past the funding of several blockchain-based companies turned out to be frauds with bogus products does not facilitate the credibility of blockchain and its applications either. In addition, there has been consistently a lot of change in the blockchain sphere regarding company or product transformation, which has led to many blockchain applications going out of service from one day to the next. This makes it even more difficult to eliminate doubts and persuasively convince the end user of the blockchain application's value. The developer's job is therefore to persuade the user to trust and value the application. In this regard users highly value comprehensive information about the product and the people behind it. Ultimately, publishing the source code is a common practice used by many successful companies and developers to significantly build trust in the application with minimal effort.

\subsection{Convey the right experience}

Developers should not overload their users with too much, but rather provide them with an intuitive user interface by relying on previous user routines. This includes creating an experience for the users by guiding them through the lifecycle of the application. Thereby developers should rigorously eliminate redundant 
steps and information from the app design. Also, trying to eliminate avoidable interfaces will strengthen the application flow and lower the kinds of abilities necessary to use the app. Especially if an application offers a payment function, we recommend integrating this function fully into the application. In doing so, the user does not have to rely on another program to access the wallet, which would mean longer engagement with your application. In addition, users should be provided with customized and individual content and information, as this would make users feel personally valued. Further, giving users the ability to try out and explore the application will help them to feel more comfortable in engaging with the application. This includes creating space for tutorials, demonstrations, and opportunities for users to familiarize themselves with the app's individual functions. However, excessively restricting the functions for the users could be patronizing. The right balance between giving guidance and allowing flexibility is therefore the key to an application's success.

\section{Discussion}

In this paper, we considered how user engagement on blockchain applications can be increased by relying on the principles of persuasive design. In developing our own blockchain-application with the help of persuasive design, we realized, that developing a usercentric blockchain app would be the key to success for future apps. Relying solely on blockchain or cryptocurrency as buzz words to generate revenue turned out to be insufficient for many applications in the past and will continue to be so in the future. Therefore, it is important for future blockchain developers to understand that which technology is used, whether it is blockchain, machine learning or IoT, is not a selling point for products and services. The decisive factor regarding an application's quality is the question of what added value it offers its users. In this case, "how" the value is added, is irrelevant. With this in mind, we would like to contribute to theoretical research in the blockchain context by answering Halaburda et al.'s [34] open questions for future research on several levels. First, using the blockchain for transactions is only useful when processes need to be automated. However, rather any kind of information is transacted over the blockchain by many applications, which could still be stored centrally without significant drawbacks. This in turn implies the answer to addressing the second research gap, which was established by Halaburda, trying to determine the extent to which different implementations of smart contracts influence their adoption. Usually, every transaction on a blockchain involves fees, and the transaction as well as its associated information on the blockchain is irreversible. Following persuasive design principles, any transaction using information which does not diminish the application's value proposition if done outside of the blockchain, does not have to be carried out through the blockchain since it would be superfluous for the users and how they perceive a particular application's persuasiveness. If such transactions are carried out centrally, it supports the user's continued control of their own data sovereignty.

We further contribute to Sadhya et al.'s [16] research on how trust in blockchain technology can be increased, giving recommendations that highlight several ways of building trust in this context. First, due to ambiguous impressions of blockchain and to quite a few scams in the past, it is necessary to provide transparency and the greatest possible flow of information to users about the application, the technology, and the people and companies behind it. Additionally, extensive customer support is an important part of increasing trust in the blockchain technology. In addition to theoretical contributions, the strength of our paper lies primarily in the practical implications, which we share in the form of six recommendations for future blockchain application developers.

\section{Conclusion}

Blockchain is one of the most disruptive technologies currently in use, bringing several new opportunities for end-user applications. Yet, the absence of persuasive design in many blockchain applications inhibits a breakthrough of blockchain technology for end-user applications. To help future blockchain application developers in their design, we developed a blockchainpowered project management tool for the creative industries, using the principles of persuasive design. By evaluating our project through a DSRP, we were able to provide seven recommendations for developers of end-user blockchain applications, summarized as (1) focus on your value proposition, (2) place only essential information on the blockchain, (3) choose the blockchain that fits your use case best, (4) leverage existing third-party services, (5) focus on comprehensive customer support, (6) be transparent and credible, and (7) convey the right kind of experience.

Through our recommendations, we contribute to research in the field of blockchain technology by providing measures that can increase user engagement for end-user blockchain applications. In addition, we contribute to persuasive design research by extending this promising framework to the use case of blockchain technology. Finally, we also provide comprehensive practical implications for a persuasive end-user blockchain design. 


\section{References}

[1] Puranam, P., O. Alexy, and M. Reitzig, "What's 'new' about new forms of organizing?", Academy of Management Review 39(2), 2014, pp. 162-180.

[2] Swartz, L., "Blockchain dreams: Imagining techno-economic alternatives after Bitcoin", Another economy is possible: culture and economy in a time of crisis 1, 2017.

[3] Schlegel, M., L. Zavolokina, and G. Schwabe, "Blockchain technologies from the consumers' perspective", (2018).

[4] Zheng, Z., S. Xie, H.-N. Dai, X. Chen, and H. Wang, "Blockchain challenges and opportunities: A survey", International Journal of Web and Grid Services 14(4), 2018, pp. 352-375.

[5] Fogg, B.J., "A behavior model for persuasive design”, (2009), 1-7.

[6] Arcos, L.C., "The blockchain technology on the music industry", Brazilian Journal of Operations \& Production Management 15(3), 2018, pp. 439-443. [7] Heywood, A., and M. Evans, "Blockchain: Disruptive technology and its effects within the music industry",

[8] Spotify Technology S.A., "Spotify Technology S.A. - Annual Report 2020”, 2020.

[9] Grześ, B., "Persuasive Design Approach. Case of Social Music Applications.”, 2013.

[10] Ichihara, Y., "Explore the Influence of Persuasive User Experience in Subscription Video on Demand Binge-Watching Consumer Behaviour", [11] Torning, K., and H. Oinas-Kukkonen, "Persuasive system design", (2009), 1-8.

[12] O'Dair, M., Distributed creativity: how blockchain technology will transform the creative economy, Springer, London, 2018.

[13] DCMS, U., "Creative industries mapping document", DCMS London, 1998.

[14] Blair, H., S. Grey, and K. Randle, "Working in film-employment in a project based industry", Personnel review 30(2), 2001, pp. 170- 185.

[15] Beck, R., J. Czepluch, N. Lollike, and S. Malone, "Blockchain-the gateway to trust-free cryptographic transactions", Springer Publishing Company (2016), 1-14.

[16] Sadhya, V., H. Sadhya, R. Hirschheim, and E. Watson, "EXPLORING TECHNOLOGY TRUST IN BITCOIN", (2018).

[17] Regner, F., N. Urbach, and A. Schweizer, "NFTs in Practice-Non-Fungible Tokens as Core Component of a Blockchain-based Event Ticketing Application", 2019.

[18] Shahab, S., and Z. Allam, "Reducing transaction costs of tradable permit schemes using Blockchain smart contracts", Growth and Change 51(1), 2020, pp. 302-308.

[19] Oinas-Kukkonen, H., "A foundation for the study of behavior change support systems", Personal and ubiquitous computing 17(6), 2013, pp. 12231235 .

[20] Schneider, H., K. Moser, A. Butz, and F. Alt, "Understanding the mechanics of persuasive system design”, (2016), 309-320.

[21] Adaji, I., and J. Vassileva, "Evaluating Persuasive Systems Using the PSD Framework.”, Citeseer (2016).

[22] Tørning, K., "Persuasive Technology Design-A Rhetorical Approach”, Springer (2008), 83-93.

[23] Kotthaus, C., T. Ludwig, and V. Pipek, "Persuasive System Design Analysis of Mobile Warning Apps for Citizens.", (2016).

[24] Langrial, S., T. Lehto, H. Oinas-Kukkonen, M. Harjumaa, and P. Karppinen, "Native Mobile Applications For Personal Well-Being: A Persuasive Systems Design Evaluation.”, (2012), 93.

[25] Oinas-Kukkonen, H., and M. Harjumaa, "Persuasive systems design: Key issues, process model, and system features", Communications of the Association for Information Systems 24(1), 2009, pp. 28.

[26] Hevner, A., and S. Chatterjee, "Design science research in information systems", In Design research in information systems. Springer, 2010, 9-22.

[27] Peffers, K., M. Rothenberger, T. Tuunanen, and R. Vaezi, "Design science research evaluation", Springer (2012), 398-410.

[28] Pries-Heje, J., R. Baskerville, and J.R. Venable, "Strategies for design science research evaluation", 2008.

[29] Peffers, K., T. Tuunanen, M.A. Rothenberger, and S. Chatterjee, "A design science research methodology for information systems research", Journal of management information systems 24(3), 2007, pp. 45-77.

[30] Webster, J., and R.T. Watson, “Analyzing the past to prepare for the future: Writing a literature review", MIS quarterly, 2002, pp. xiii-xxiii.

[31] Haigh, T., F. Breitinger, and I. Baggili, "If i had a million cryptos", Springer (2018), 45-65.

[32] Mills, S.B., “IBM's Blockchain Design Principles", Medium.

[33] Wieck, M., and J. Cuomo, "Blockchain as a force for good - Five principles to build trust and value", 2019. https:/www.ibm.com/downloads/cas/AGL5ZWLN

[34] Halaburda, H., N. Levina, and M. Semi, "Understanding smart contracts as a new option in transaction cost economics", (2019). 